\title{
Contrôle des composés indésirables dans les huiles végétales et mise en place d'observatoires
}
F. LACOSTE
H. LECHAT
X. PAGES
J.N. ARNAUD
E. BRENNE
B. SOULET
B. CAMISULI
C. BIROT
S. FAZEUILH
J. ESCABASSE

Iterg, Institut des Corps Gras,

$<$ f.lacoste@iterg.com>

www.iterg.com
Rue Monge, 33600 Pessac, France

\begin{abstract}
The presence of undesirable compounds in vegetable and animal oils and fats may have many different origins such as environment, crop protection, production processes, transport and storage, packaging and industrial utilisation like frying. Although the potential toxicity of most of these undesirable compounds is real, poisoning risks are rather limited due to the efficient elimination during oil-refining steps, careful conditioning, choice of efficient packaging and industrial quality control management. For some undesirable compounds representative of the major origins listed above, the analytical methods used (sensitivity, precision), the existing regulations (EU and Codex) and an idea of their content in fats and oils are presented. Main conclusions are:

- For most of the undesirable compounds possibly found in oils and fats, standardised test methods do exist or are in progress.

- Results presented prove that oil-refining steps are efficient for elimination of most undesirable compounds.

- Oils and fats present in general low level of undesirable compounds such as lead, cadmium, hexane, PAHs, pesticide residues or mycotoxins.

- In order to get reliable and up to date data and to check the conformity of the products regarding the present regulations, the creation of survey plans on potential contaminants such as pesticide residues and benzo[a]pyrene is suggested for refined oils and for cold pressed oils, lead and ochratoxin $A$ have to be added to the list.
\end{abstract}

Key words: vegetable oil, contaminants, pesticide residues, polycyclic aromatic hydrocarbons, refining, survey plan

\section{Origine des contaminants}

Les principaux facteurs influant sur la présence de contaminants dans une huile végétale sont différents d'une étape à l'autre du procédé de transformation. Ainsi, au niveau de la culture, I'environnement, la protection des cultures et les conditions climatiques ont un effet direct sur la présence potentielle de métaux lourds tels que le plomb ou le cadmium, de résidus phytosanitaires ou de mycotoxines (figure 1). Par le biais de la contamination de l'environnement (air, eau, sol...), des traces de polychlorobiphényls et de dioxines peuvent être retrouvées dans les matières grasses, en particulier dans les graisses animales et les huiles de poisson.

Au cours du stockage et du transport, les graines oléagineuses peuvent être en contact avec des insecticides destinés au traitement des silos, et du matériel de transport.

Le séchage des matières premières (graines ou fruits oléagineux) puis l'utilisation de solvants lors de l'extraction de l'huile, sont sources respectivement d'hydrocarbures aromatiques polycycliques et de traces d'hexane résiduel. Le transport de l'huile brute présente également un risque d'éventuelle contamination par des huiles minérales ou d'autres dérivés du pétrole, provenant de précédentes cargaisons.

Au cours des procédés de raffinage et d'hydrogénation, la formation d'acides gras en configuration trans est à craindre. L'utilisation de cataly-

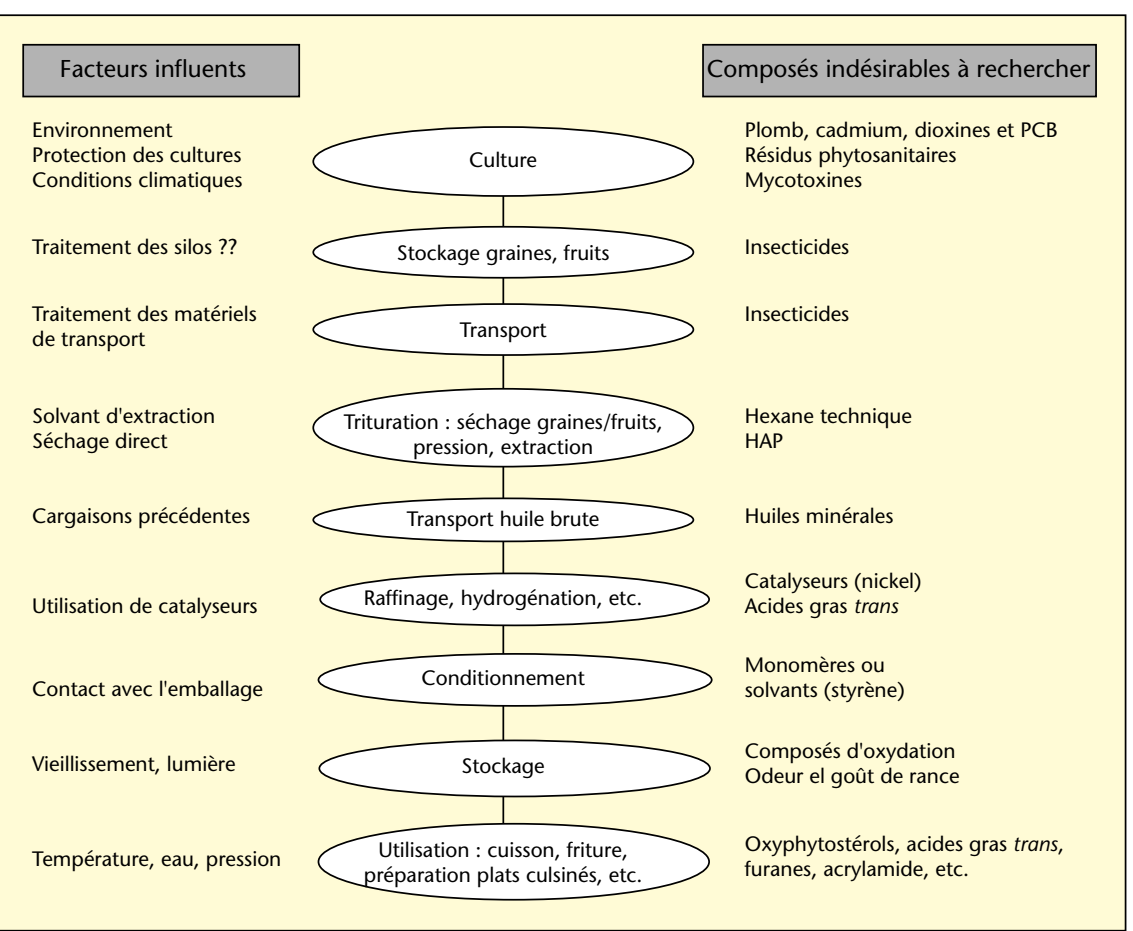

Figure 1. Suivi du produit au cours du procédé de transformation.

seur à base de nickel lors de l'hydrogénation des huiles nécessite de s'assurer de son élimination. Le conditionnement des huiles et graisses les met en contact avec des matières plastiques dont certains monomères ou solvants résiduels liposolubles peuvent migrer dans le produit (styrène, etc.). 
Enfin, lors de la conservation puis de leur utilisation en cuisson ou friture, les matières grasses sont sujettes à des dégradations de leurs constituants sous l'action de paramètres tels que la lumière, l'oxygène ou la température.

\section{Méthodes d'analyse et réglementation}

\section{Détermination des métaux}

La détermination des traces métalliques dans les huiles et graisses se fait par dosage direct par absorption atomique électrothermique [1-4] ou émission atomique (torche à plasma) après dilution de l'échantillon dans un solvant organique. Des méthodes normalisées existent pour le cuivre, le fer, le nickel (ISO 8294), le cadmium (ISO 15774) et le plomb (ISO 12193). Des limites bien supérieures aux limites de quantification des méthodes existantes sont fixées par le Codex Alimentarius pour le plomb et l'arsenic (tableau 1). II en est de même pour les valeurs fixées par la réglementation française pour le nickel et le chrome, ainsi que pour celle fixée par la Commission européenne pour le plomb (CE 466/2001).

\section{Détermination des solvants : hexane, solvants halogénés}

La détermination des solvants comme I'hexane ou les solvants halogénés dans les huiles et graisses est réalisée en générant un espace de tête et en l'analysant par chromatographie en phase gazeuse avec un détecteur à ionisation de flamme.

Afin de diminuer la limite de quantification de la norme ISO 9832 de 10 mg/kg à moins de $1 \mathrm{mg} / \mathrm{kg}$ [5], qui est la valeur fixée au niveau européen (97/60/CE), une révision de la norme est en cours (tableau 2). Les modifications techniques proposées par la France [6], sont basées sur l'injection d'un volume gazeux plus important ainsi que sur l'utilisation d'une colonne de dimension adaptée (diamètre de $0,53 \mathrm{~mm}$ ).

\section{Détermination des mycotoxines}

L'analyse des mycotoxines a été récemment décrite par Papachristou et Markaki [7]. L'ochratoxine A y est extraite par un mélange de méthanol/eau (80:20) puis purifiée sur une cartouche de support immuno-enzymatique, I'analyse étant effectuée par HPLC avec un détecteur à fluorescence. L'aflatoxine B1 est extraite avec un protocole similaire mais il est nécessaire de prévoir une étape intermédiaire de dérivation préalablement à son analyse par HPLC afin d'être détectée avec le même détecteur. Les limites de détection sont respectivement de 5 et $56 \mathrm{ng} / \mathrm{kg}$ pour l'ochratoxine A et

Tableau 1. Comparaison des limites de quantification des méthodes normalisées pour la détermination des métaux toxiques et des limites en vigueur (normes commerciales, règlements nationaux ou européens).

\begin{tabular}{|lccccc|}
\hline Métaux & Cd & Pb & Ni & As & Cr \\
\hline Limite de quantification $(\mathrm{mg} / \mathrm{kg})$ & 0,002 & 0,010 & 0,015 & 0,010 & 0,005 \\
Réglementation $(\mathrm{mg} / \mathrm{kg})$ & - & 0,1 & 0,2 & 0,1 & 0,05 \\
& & UE \& Codex & France & Codex & France \\
\hline
\end{tabular}

Tableau 2. Limites de quantification des méthodes normalisées ou en cours d'étude pour la détermination de I'hexane.

\begin{tabular}{|lccc|}
\hline Méthode hexane & ISO 9832 & NF T 60-257 & ITERG \\
\hline Limite de quantification $(\mathrm{mg} / \mathrm{kg})$ & 10 & 2 & 0,3 \\
\hline
\end{tabular}

Tableau 3. Comparaison des limites de quantification des méthodes publiées par Papachristou et Markaki pour la détermination des mycotoxines avec les limites en vigueur (réglementation française).

\begin{tabular}{|lccc|}
\hline Mycotoxines & Aflatoxine B1 & Ochratoxine & Zéaralénone \\
& & A & \\
\hline Limite de détection $(\mu \mathrm{g} / \mathrm{kg})$ & 0,056 & 0,005 & $?$ \\
Réglementation $(\mu \mathrm{g} / \mathrm{kg})$ & 5 (huile) & - & 200 (huile) \\
& France $[8]$ & & France [8] \\
\hline
\end{tabular}

I'aflatoxine B1 dans I'huile d'olive, ce qui est bien inférieur comme niveau à celui fixé par la réglementation française pour l'aflatoxine B1 (tableau 3).

\section{Détermination des résidus de pesticides}

Dans le cadre de la mise en place d'un plan de surveillance portant sur la qualité sanitaire des produits de la filière oléagineuse, un groupe de travail animé par le Cetiom et I'Iterg, a entrepris de développer une méthode d'analyse des résidus de pesticides dans les graines et les huiles en vue de sa normalisation (Afnor et CEN).

Ce groupe qui réunit environ 25 laboratoires d'analyse privés et publics, a procédé à une compilation des méthodes les plus performantes à l'issu d'un essai circulaire organisé en 2004.

Les résidus étudiés, au nombre de 16 , ont été choisis en fonction des travaux préliminaires de I'Iterg sur les huiles issues de la trituration et avant raffinage (tableau 4). La méthode ainsi élaborée sera ensuite validée par une série d'essais circulaires.

L'Iterg travaille depuis plus d'une dizaine d'années sur l'analyse des résidus de pesticides dans les huiles végétales et a proposé en 1997 une méthode d'extraction et de purification. Cette méthode met en œuvre des cartouches d'adsorbant et permet ainsi de réduire les quantités de solvants utilisées [9]. Les limites de quantification obtenues sont en accord avec les valeurs fixées par la réglementation européenne pour les graines oléagineuses (figure 2). Rappelons qu'il n'existe pas de limites régle- mentaires pour les huiles végétales au niveau européen.

\section{Détermination des hydrocarbures aromatiques polycycliques (HAP)}

Le dosage des hydrocarbures aromatiques polycycliques dans les huiles nécessite le plus souvent une purification sur alumine, silice greffée en C18 ou par transfert de charge (DACC), suivie d'une analyse par HPLC/fluorescence. Les méthodes normalisées ou en cours de l'être sont les suivantes:

- ISO 15032 : Détermination de la teneur en benzo[a]pyrène par HPLC/fluorescence [10]. - ISO/DIS 15753: Détermination des hydrocarbures aromatiques polycycliques, méthode développée par l'Iterg et basée sur une extrac-

Tableau 4. Liste des résidus de pesticides étudiés par le groupe de travail français sur les méthodes d'analyse dans les graines et les huiles.

\begin{tabular}{|ll|}
\hline \multicolumn{2}{|c|}{ Résidus organochlorés et dicarboximides } \\
Endosulfan & Heptachlore \\
Vinclozoline & Lindane \\
\multicolumn{2}{|c|}{ Résidus organophosphorés } \\
Chlorpyriphos éthyl & Chlorpyriphos méthyl \\
Diazinon & Dichlorvos \\
Fenitrothion & Malathion \\
Parathion méthyl & Pyrimiphos méthyl \\
\multicolumn{2}{|c}{ Résidus pyréthrinoïdes } \\
Deltaméthrine & Cyfluthrine \\
Cyperméthrine & lambda-Cyhalothrine \\
\hline
\end{tabular}




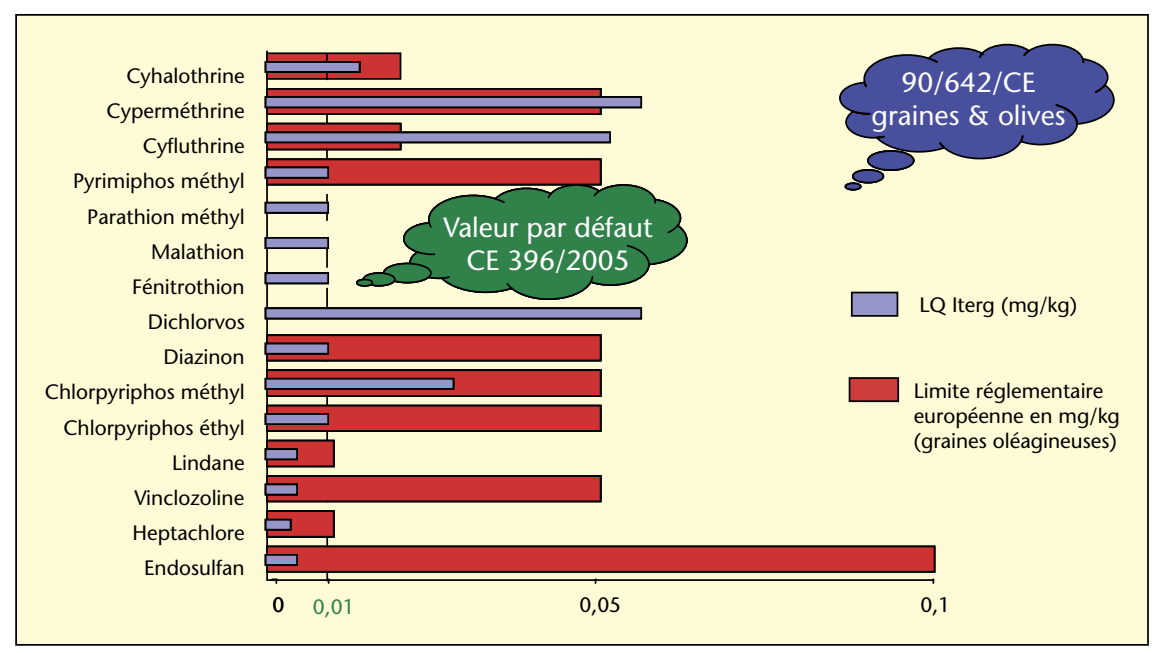

Figure 2. Comparaison des limites de quantification des résidus de pesticides dans les huiles (méthode Iterg) avec les limites réglementaires européennes pour les graines oléagineuses.

Tableau 5. Comparaison des limites de quantification du benzo[a]pyrène des méthodes normalisées ou en cours de normalisation pour la détermination des hydrocarbures aromatiques polycycliques dans les huiles.

\begin{tabular}{|lccc|}
\hline Méthode & ISO 15302 & pr ISO 15753 & DACC \\
\hline Limite de quantification $\mathrm{BaP}(\mu \mathrm{g} / \mathrm{kg})$ & 0,1 & 0,2 & 0,1 \\
\hline
\end{tabular}

tion avec un mélange acétonitrile/acétone suivie de purifications sur cartouches de silice greffée C18 puis Florisil, et détermination par HPLC/fluorescence [11].

- ISO/AWI 24054 (projet) : Détermination des hydrocarbures aromatiques polycycliques par CPG et détection par spectrométrie de masse, méthode basée sur une étape de saponification, suivie d'une purification sur gel de silice puis d'une analyse par CPG/MS avec dilution isotopique.

- ISO/AWI 22959 (projet) : Détermination des hydrocarbures aromatiques polycycliques par chromatographie de complexe donneuraccepteur d'électron (DACC), méthode basée sur une double chromatographie HPLC en ligne, la première sur colonne de type " donneur-accepteur » permettant d'isoler les $\mathrm{HAP}$, la seconde sur silice greffée C18 assurant I'analyse des HAP [12].

Le règlement européen CE 208/2005 considère le benzo(a)pyrène comme un marqueur des HAP et fixe une teneur limite de $2 \mu \mathrm{g} / \mathrm{kg}$ pour celui-ci [13]. Les méthodes développées permettent d'atteindre ce niveau de sensibilité (tableau 5) du moment que l'échantillon ne présente pas de pics interférents.

\section{Détermination des dioxines et polychlorobiphényls (PCB)}

L'une des premières méthodes décrites pour la détermination des polychlorodibenzodioxines (PCDD) et des polychlorodibenzofuranes
(PCDF) l'a été par l'Agence pour la protection de l'environnement américaine (EPA) : méthode EPA 1613 [14]. Elle est basée et spectrométrie de masse haute résolution en dilution isotopique $\left({ }^{13} \mathrm{C}_{12}\right.$-PCDD et ${ }^{13} \mathrm{C}_{12}$ $P C D F)$ après purification à l'aide de différents adsorbants. Le calcul de la teneur totale OMSTEQ en $\mathrm{pg} / \mathrm{g}$ est ensuite réalisé en tenant compte des facteurs d'équivalence toxicologique. Cette technique fournit une sensibilité très élevée, de l'ordre de 0,2 ng/ $\mathrm{kg}$ (ou pg/g).

Le règlement CE 2375/2001 qui devrait bientôt être modifié pour y intégrer les PCB-type dioxines [15], fixe des teneurs très faibles en PCDD et PCDF pour certains produits comme les huiles végétales et graisses animales (tableau 6).

\section{Efficacité du raffinage}

Outre l'obtention d'une huile neutre au niveau organoleptique, le raffinage permet également

Tableau 6. Limites réglementaires en $P C D D$ et $P C D F$ par $g$ de matière grasse.

\begin{tabular}{|lc|}
\hline Graisse animale & \\
de ruminants & 3 \\
de volaille & 2 \\
saindoux & 1 \\
mélange & 2 \\
Huile végétale & 0,75 \\
Huile de poisson (alimentation humaine) & 2 \\
\hline
\end{tabular}
sur une analyse par couplage chromatographie exprimées en pg (10-12 g) d'équivalent toxique (OMS) d'éliminer un certain nombre de composés indésirables et contaminants. Depuis 2002, I'Iterg s'est doté d'un pilote de raffinage d'une capacité de 300-1 $000 \mathrm{~kg}$ par batch, permettant de réaliser des opérations de raffinage physique ou chimique telles que dans I'industrie.

Cet outil a été utilisé pour divers programmes d'étude visant à évaluer l'influence des conditions opératoires sur l'élimination des résidus de pesticides (figures 3 et 4).

En raffinage chimique, le dichlorvos est intégralement éliminé lors de la neutralisation alors qu'une étape de désodorisation menée à $220^{\circ} \mathrm{C}$ est nécessaire pour aboutir au même résultat pour le malathion et le fénitrothion, pour des taux de contamination initiale de l'ordre de $0,5 \mathrm{mg} / \mathrm{kg}$. Le pyrimiphos méthyl est lui éliminé au cours de l'étape de décoloration. Le raffinage physique fournit des résultats équivalents mais la température de la désodorisation étant plus élevée, autour de $240{ }^{\circ} \mathrm{C}$, il ne reste plus aucun résidu à la fin de cette étape.

L'élimination des hydrocarbures aromatiques des huiles de pépins de raisin a été étudiée il y a quelques années à l'Iterg [11]. Les résultats ont prouvé que, pour des taux de contamination de l'ordre de $600 \mu \mathrm{g} / \mathrm{kg}$ en HAP totaux, tous les HAP lourds (plus de 4 cycles aromatiques condensés) et la majeure partie des HAP légers étaient éliminés durant l'étape de décoloration lorsque du charbon actif était ajouté aux terres décolorantes. L'étape de désodorisation permet de s'affranchir des HAP légers restants.

Des études complémentaires sont en cours sur cette thématique d'élimination des pesticides et HAP lors du raffinage. Des taux de contamination élevés sont testés ainsi que des traitements particuliers : adsorbants choisis, traitement séquentiel, etc. L'influence des conditions opératoires est en outre examinée.

\section{Plans de surveillance}

La maitrise des contaminants potentiels passe par l'action préventive. C'est la raison pour laquelle l'Iterg participe activement à la mise en place de plan de surveillance des produits mis en marché. Le principe du plan de surveillance réside dans la mise en commun des données d'autocontrôle des industriels d'une profession, avec un retour de l'information globale sous forme de statistiques. Ses principaux intérêts sont de fournir une connaissance du niveau et de l'évolution d'une contamination spécifique, de prévoir et anticiper des événements préjudiciables, d'établir un dossier consistant et actualisé pour affronter une éventuelle situation de crise.

La stratégie généralement adoptée passe par : - le choix des contaminants à surveiller en priorité suite à un travail d'enquête ; 


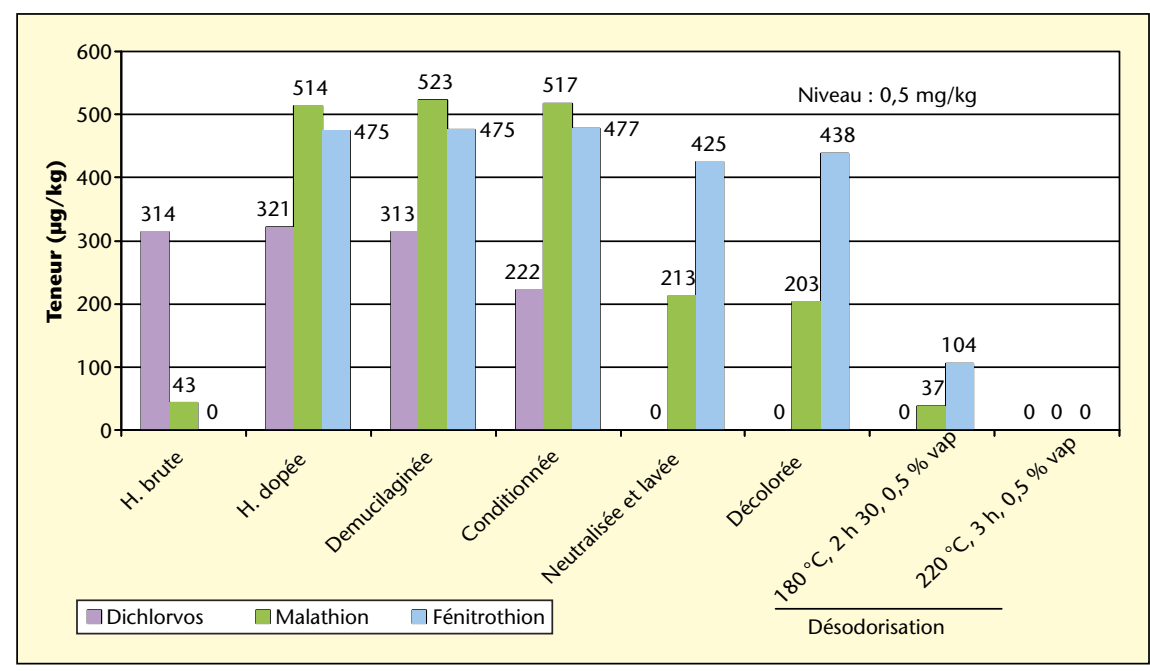

Figure 3. Élimination des résidus de pesticides d'une huile brute artificiellement contaminée en dichlorvos, malathion et fénitrothion, au cours d'un raffinage chimique sur le pilote de l'Iterg (1 tonne).

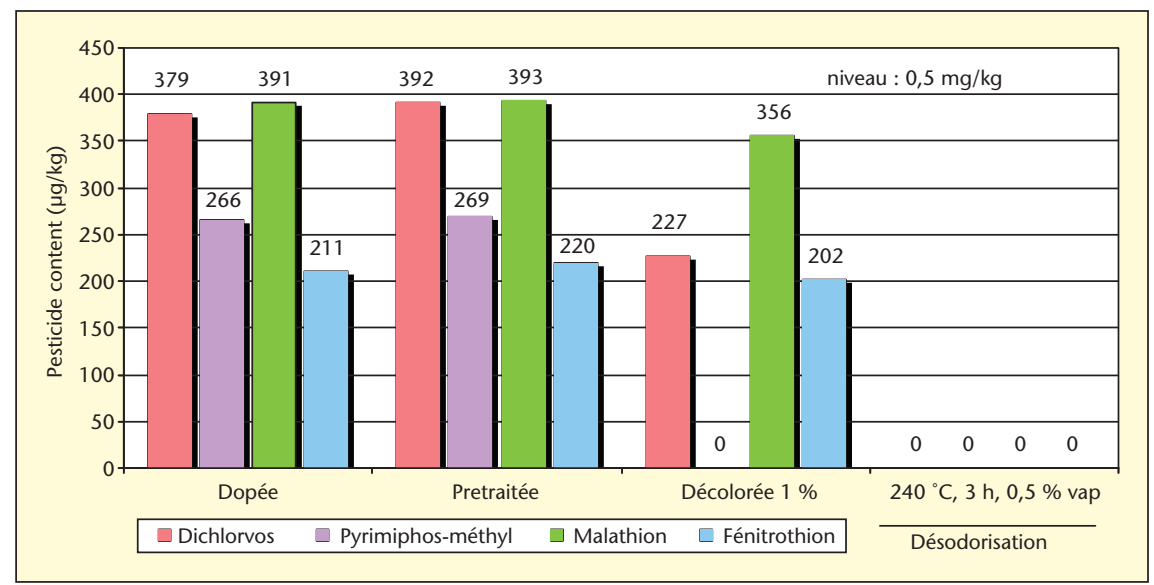

Figure 4. Elimination des résidus de pesticides d'une huile brute artificiellement contaminée en dichlorvos, pyrimiphos méthyl, malathion et fénitrothion, au cours d'un raffinage physique (25 kg).

- la rédaction d'une convention de confidentialité avec les adhérents ;

- la garantie de la fiabilité et l'homogénéité des données obtenues.

\section{Travaux d'enquête}

Un état des lieux réalisé récemment sur l'éventuelle contamination de l'huile d'olive vierge par un certain nombre de composés [16], tels que les solvants chlorés, les hydrocarbures aromatiques volatils, les hydrocarbures aromatiques polycycliques, les métaux toxiques, les résidus de pesticides et les mycotoxines, a montré que les résultats disponibles dans la littérature scientifique sont, en majorité, conformes à la réglementation en vigueur. Cependant, compte tenu des réglementations existantes, la mise en place de plans de surveillance sur des contaminants tels que les résidus de pesticides, le benzo[a]pyrène, le plomb, et l'ochratoxine A est recommandée. brutes de tournesol et colza (64 échantillons examinés).
Les travaux d'enquête réalisés par l'Iterg au cours des deux dernières années sur l'état sanitaire des huiles brutes de tournesol et de colza ont montré que le raffinage était nécessaire pour éliminer les résidus de pesticides des huiles brutes. En effet, très peu d'échantillons sont exempts de résidu $(<0,01 \mathrm{mg} / \mathrm{kg})$. La teneur globale en résidus de pesticides est en moyenne comprise entre 0,1 et $0,25 \mathrm{mg} / \mathrm{kg}$, avec des teneurs maximales de l'ordre de 1 $\mathrm{mg} / \mathrm{kg}$ (figure 5). Les résidus identifiés le plus fréquemment sont le pyrimiphos méthyl, le malathion et le dichlorvos.

Lors de ces enquêtes, l'analyse des hydrocarbures aromatiques polycycliques a mis en évidence la présence de HAP légers (figure 6) alors que les teneurs globales en HAP lourds sont minimes $(<5 \mu \mathrm{g} / \mathrm{kg})$. Toutes les huiles analysées sont conformes à la réglementation européenne qui fixe une teneur maximale autorisée de $2 \mu \mathrm{g} / \mathrm{kg}$ en benzo[a]pyrène dans les huiles et graisses destinées à l'alimentation humaine. Une enquête récente publiée par la Commission européenne [17] est moins optimiste pour I'huile de grignons d'olive puisque plus de $60 \%$ des échantillons analysés contiennent plus de $2 \mu \mathrm{g} / \mathrm{kg}$ (figure 7).

\section{Plans de surveillance en cours dans le domaine des huiles végétales}

Deux plans de surveillance ont été mis en place dans le domaine des huiles végétales, le premier est centré sur le suivi des résidus de pesticides dans les huiles d'olive vierges, le second concerne l'ensemble de la filière oléagineuse. Ce dernier est issu d'un travail commun des deux centres techniques, l'Iterg et le Cétiom, en collaboration avec l'Onidol, afin de suivre dans les graines, les huiles et les tourteaux, un certain nombre de contaminants (tableau 7). Plus de 700 données ont été collectées en un

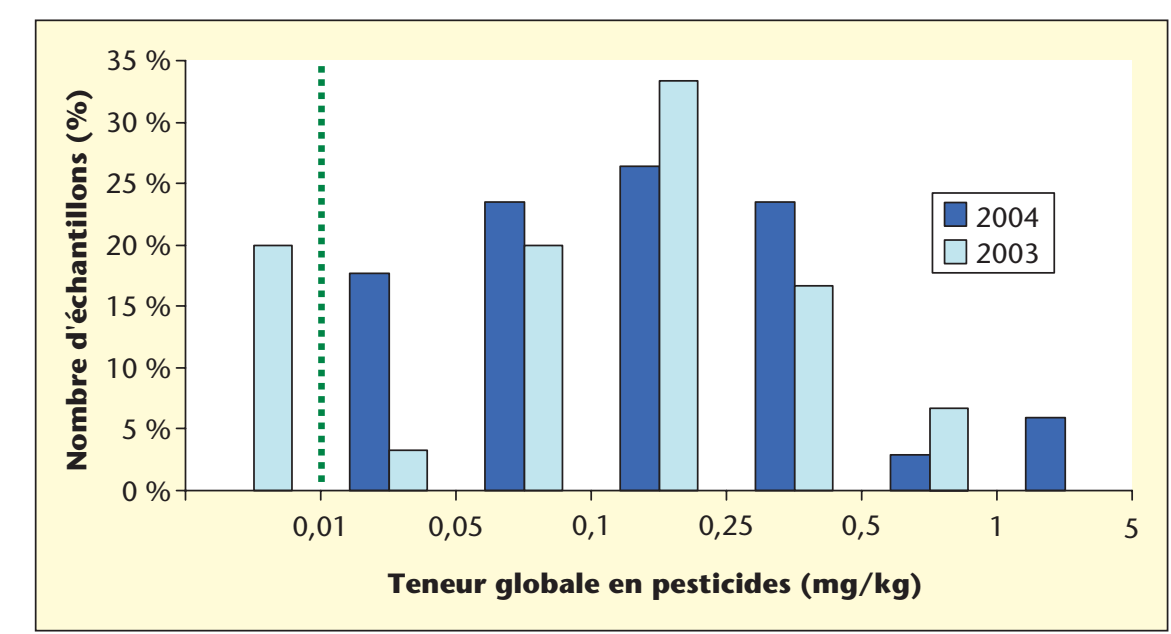

Figure 5. Résultats des enquêtes menées en 2003 et 2004 sur la présence des résidus de pesticides dans les huiles 


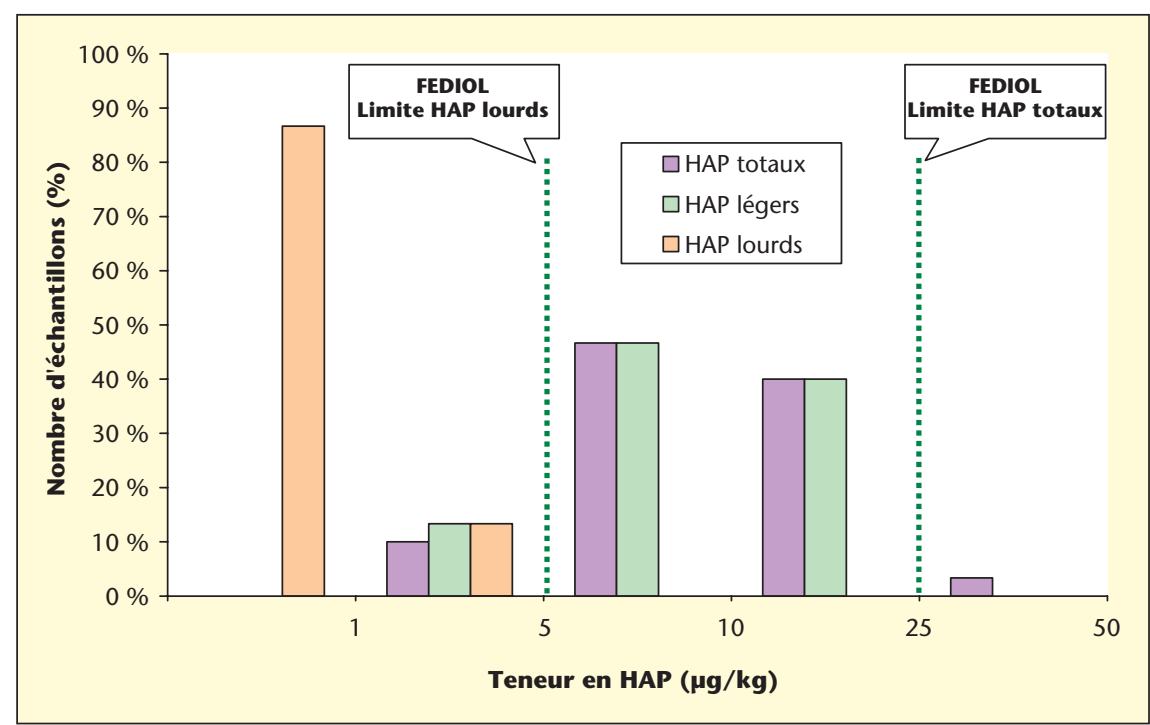

Figure 6. Résultats des enquêtes menées en 2003 et 2004 sur la présence des hydrocarbures aromatiques polycycliques dans les huiles brutes de tournesol et colza (64 échantillons examinés).

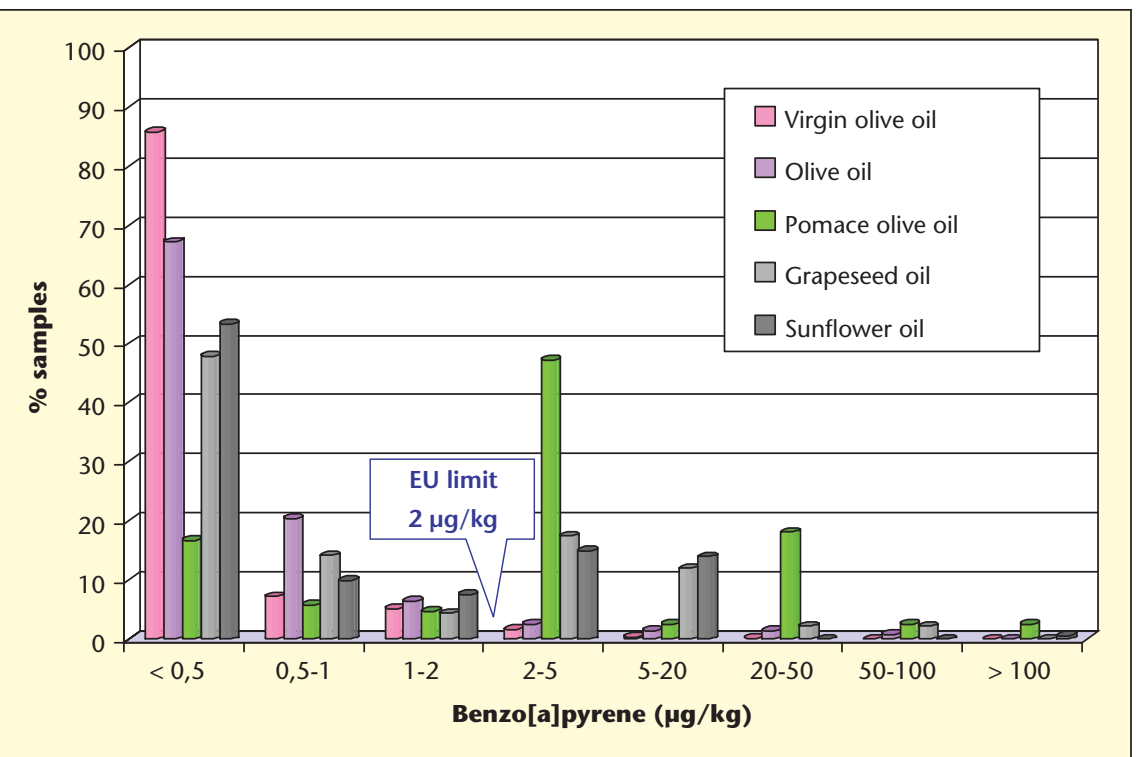

Figure 7. Résultats de l'enquête de la Tâche SCOOP sur la présence de benzo[a]pyrène dans les huiles végétales en Europe.

Tableau 7. Liste des contaminants prioritaires à rechercher dans les graines, huiles et tourteaux de colza, tournesol, soja.

\begin{tabular}{|c|c|c|c|}
\hline Contaminants & Graines & Tourteaux & Huiles \\
\hline Mycotoxines & Aflatoxine B1 & & \\
\hline Résidus de pesticides & & & $\begin{array}{l}\text { Organochlorés- } \\
\text { organophosphorés- } \\
\text { pyréthrinoides }\end{array}$ \\
\hline Métaux lourds & Cadmium-plomb & & \\
\hline Bactéries & & Salmonelles & \\
\hline Autres & & & HAP \\
\hline
\end{tabular}

an d'existence de ce plan de surveillance " oléagineux ».

\section{Conclusion}

Même si les huiles et matières grasses contiennent de faibles teneurs en composés indésirables tels que le plomb, cadmium, hexane, HAP ou des résidus de pesticides, le raffinage permet de s'affranchir d'une grande partie d'entre elles. Une mise au point de l'influence des conditions opératoires du raffinage sur l'élimination de ces contaminants est toutefois nécessaire afin d'améliorer la maîtrise de cette problématique pour le raffinage.

Afin de disposer de données fiables et actualisées permettant de vérifier le respect des réglementations en vigueur, la mise en place de plans de surveillance est vivement conseillée, pour des contaminants comme :

- les résidus de pesticides et le benzo[a]pyrène, pour les huiles brutes et raffinées ;

- les résidus de pesticides, le benzo[a]pyrène, le plomb, et l'ochratoxine A pour les huiles vierges.

\section{RÉFÉRENCES}

1. LACOSTE F, CASTERA A, LESPAGNE J. Dosage des métaux toxiques dans les corps gras : cadmium, plomb, étain, arsenic et chrome (méthodes et applications). Rev Franç Corps Gras 1993 ; 40(1/2) : 19-31.

2. NF EN ISO 8294. Corps gras d'origines animale et végétale - Détermination de la teneur en cuivre, fer et nickel - Méthode par spectrométrie d'absorption atomique avec four en graphite. 1999.

3. EN ISO 15774. Corps gras d'origines animale et végétale - Détermination de la teneur en cadmium par spectrométrie d'absorption atomique à four graphite. 2001.

4. NF EN ISO 12193. Corps gras d'origines animale et végétale-Détermination de la teneur en plomb - Méthode par spectrométrie d'absorption atomique avec four en graphite. 2004.

5. ISO 9832. Corps gras d'origines animale et végétale - Dosage de l'hexane technique résiduel. 2003.

6. LACOSTE F, BRENNE E, COUSTILLE JL. Amélioration des méthodes de dosage de traces $\mathrm{d}^{\prime}$ hexane résiduel dans les huiles végétales raffinées. OCL $2001 ; 8$ : 167-73.

7. PAPACHRISTOU A, MARKAKI P. Determination of ochratoxin A in virgin olive oils of Greek origin by immunoaffinity column clean-up and high-performance liquid chromatography. Food Addit Contam 2004 ; 21(1) : 85-92.

8. Note de la DGCCRF n5662 du 12.12.1990, basé sur un avis du Conseil Supérieur d'Hygiène Publique de France, LAMY-DEHOVE, étude 215, paragraphe 338. 
9. RAOUX R, COUSTILLE JL, RAMBAUD S. Analyse des pesticides organochlorés, organophosphorés et des polychlorobiphényls dans les corps gras. Méthode d'extraction et de purification multicartouches. OCL $1997 ; 4(1)$ : 71-80.

10. NF ISO 15302. Corps gras d'origines animale et végétale - Détermination de la teneur en benzo[a]pyrène - Méthode par chromatographie en phase liquide à haute performance à polarité de phase inversée. 1998.

11. LACOSTE F, RAOUX R, DUBOIS D, SOULET $B$. Problématiques des hydrocarbures aromatiques polycycliques dans les corps gras. $\mathrm{OCL}$ 2003 ; 10(4) : 287-95
12. VAN STIJN F, KERKHOFF MAT, VANDEGINSTE BGM. Determination of polycyclic aromatic hydrocarbons in edible oils and fats by on-line donor-acceptor complex chromatography and high-performance liquid chromatography with fluorescence detection. I Chromatography $A$ $1996 ; 750: 263-73$.

13. Règlement européen CE 208/2005 du 4 février 2005 modifiant le règlement CE 466/2001 en ce qui concerne les hydrocarbures aromatiques polycycliques.

14. Méthode EPA. 1613 (1994) : http ://www.epa. gov/waterscience/methods/1613.pdf.
15. Règlement CE. 2375/2001 du 29 novembre 2001 modifiant le règlement CE 466/2001 en ce qui concerne les PCDD et PCDF.

16. LACOSTE F, SOULET B, ARNAUD JN, BRENNE E, LECHAT H. Contrôle de la sécurité sanitaire de l'huile d'olive vierge. OCL 2004 ; $11(3)$ : 210-6.

17. Scoop Task EU. (Oct 2004), http ://europa.eu. int/comm/food/food/chemicalsafety/contaminants/scoop_3-2-12_final_report_pah_en.pdf. 